\title{
Measuring Safety Through the Distance Between System States with the RiskSOAP Indicator
}

\author{
Mara Mikela Chatzimichailidou \\ University of Cambridge, Engineering Design Centre, Trumpington Street, Cambridge, CB2 \\ 1PZ, United Kingdom \\ Tel: +44 4790 276975. E-mail: mmc60@can.ac.uk \\ Nektarios Karanikas \\ Amsterdam University of Applied Sciences, Faculty of Technology, Weesperzijde 190, \\ Amsterdam, 1097 DZ, the Netherlands \\ Ioannis Dokas \\ Democritus University of Thrace, Polytechnic School, Vassilissis Sofias 12, Xanthi, 6710, \\ Greece
}

doi:10.5296/jss.v2i2.10436ＵRL: http://dx.doi.org/10.5296/jss.v2i2.10436

\begin{abstract}
Modern engineering systems are complex socio-technical structures with a mission to offer services of high quality, while in parallel ensuring profitability for their owners. However, practice has shown that accidents are inevitable, and the need for the use of systems-theoretic tools to support safety-driven design and operation has been acknowledged. As indicated in accident investigation reports, the degradation of risk situation awareness (SA) usually leads to safety issues. However, the literature lacks a methodology to compare existing systems with their ideal composition, which is likely to enhance risk SA. To fill this gap, the risk SA provision (RiskSOAP) is a comparison-based methodology and goes through three stages: (1) determine the desired/ideal system composition, (2) identify the as-is one(s), (3) employ a comparative strategy to depict the distance between the compared units. RiskSOAP embodies three methods: STPA (System Theoretic Process Analysis), EWaSAP (Early Warning Sign Analysis) and dissimilarity measures. The practicality, applicability and generality of RiskSOAP is demonstrated through its application to three case studies. The purpose of this work is to suggest the RiskSOAP indicator as a measure for safety in terms of the gap between system design and operation, thus increasing system's risk SA. RiskSOAP can serve as a criterion for planning system modifications or selecting between alternative systems, and can support the design, development, operation and maintenance of safe systems.
\end{abstract}

Keywords: Dissimilarity measures; Risk situation awareness, RiskSOAP, Socio-technical systems, STPA, EWaSAP 


\section{Introduction}

Complex socio-technical systems consist of many parts, controlled by human or automated agents spread throughout different hierarchical levels. In such systems safety is one of the primary goals, denoting that agents that control a part of the system should be enabled to perceive and comprehend threats and vulnerabilities, as well as projecting what they may entail in concordance with the system characteristics and mission. In essence, they should bear risk-focused situation awareness (SA). This presupposes that an agent should be offered an indication of system states variability in order to update his/her mental model and adjust system processes accordingly. As various authors point out, one of the most critical high-level risks is the large gap between work-as-imagined and work-as-done (Woltjer et al., 2015; Blandford et al., 2014). Hence, in order to maintain the safety levels for which the system was originally planned, controllers must be aware of the distance between system design and operation. In this setting, the risk SA provision (RiskSOAP) is operationalised through a quantification of the differences of various system versions in regard to safety, and in this way supporting the SA of its agent(s) (Chatzimichailidou, 2016). RiskSOAP may be increased or decreased by including or excluding, upgrading, downgrading or maintaining system parts and elements, or their properties, throughout the system's lifecycle.

The paper in hand presents the RiskSOAP methodology and comprises a summary of previous publications as a means to provide the reader with an overall view and a comprehensive demonstration of its applicability. This methodology consists of three stages: (1) determine the composition of the ideal - desired system, (2) identify the as-is system composition(s), (3) employ a comparative strategy to depict the distance between the ideal and as-is systems. The aforesaid stages are performed by employing three methods: STPA (System Theoretic Process Analysis) (Leveson, 2011), EWaSAP (Early Warning Sign Analysis) (Dokas et al., 2013), which extends STPA, and dissimilarity measures. The application of the RiskSOAP methodology leads to an indicator that measures the RiskSOAP and renders the latter as a measure for safety, in terms of the distance between the optimal design and the current system state, as well as between system states at different time points. The proposed methodology is demonstrated through three case studies: (1) the “ACROBOTER" robotic platform (Stepan et al., 2009), the system(s) operated in the Überlingen mid-air collision accident (Johnson, 2004; BFU, 2002) and a road tunnel (Chatzimichailidou and Dokas, 2016).

In order to avoid any confusion between the RiskSOAP methodology and the existing SA measurement techniques (Chatzimichailidou, 2016) the authors emphasise that SA measurement techniques attempt a direct measurement of SA, which is out of the scope of the RiskSOAP methodology. RiskSOAP is grounded on a comparison between two (or more) versions of a complex socio-technical system that differ in the elements and characteristics which affect safety, thus it enhances risk awareness of the system controllers (Chatzimichailidou and Dokas, 2016). Thus, neither a direct measurement nor an assessment of the SA shape the primary goal of this research work because the 'measured substance' is different compared to the existing SA measurement techniques. The quantification we propose in this paper allows the analyst to evaluate existent systems or alternative system 
designs and, possibly, enforce controls that will maximise system safety (Chatzimichailidou and Dokas, 2015).

\section{The RiskSOAP Methodology}

Figure 1 shows the sequence of the steps of the RiskSOAP methodology. The methods that comprise the RiskSOAP methodology are presented in brief below.

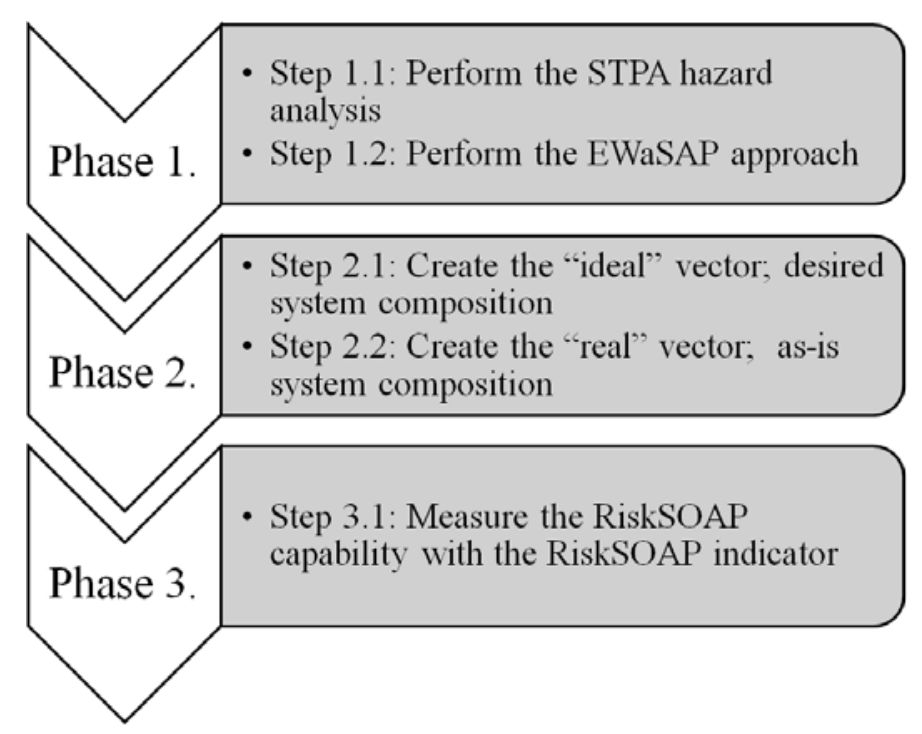

Figure 1. The RiskSOAP methodology

\subsection{STAMP and STPA}

Leveson's (2011) Systems-Theoretic Accident Model and Processes (STAMP) is an accident model which is based on systems control theory and extends the traditional analytic reduction and reliability theories. It mainly advocates that accidents involve a complex dynamic process, so they are not simply chains of events and component failures. For this reason, STAMP theory views safety as an emergent property that arises when system components interact with each other within their larger environment.

STPA is a hazard analysis technique that encapsulates the principles of the STAMP model. Because STPA is a top-down approach to system safety, it can be used to generate safety requirements and constraints of existing systems or systems early in the development phase (Leveson, 2011). STPA is a rigorous method through which the analyst identifies inadequate control actions and examines scenarios or paths to accidents instead of calculating probabilities of failures and events or estimating severity of outcomes (Leveson, 2011). STPA also identifies causal factors not fully handled by traditional hazard analysis methods, such as software errors, component interactions, decision-making flaws, inadequate coordination and conflicts among multiple controllers, and poor management and regulatory decision-making (Leveson, 2015). Safety is, thus, treated as a dynamic control problem, rather than a component reliability problem. 


\subsection{EWaSAP}

EWaSAP is an add-on to STPA (Leveson, 2015; Dokas et al., 2013) and its aim is to provide a structured method for the identification of early warning signs required to update mental models of system agents. Under this approach, EWaSAP introduces an additional type of control action, the awareness action. An awareness control action is required from a controller who must provide warning messages and alerts to other controllers inside or outside the system boundaries whenever data indicating the presence of threats or vulnerabilities is perceived and comprehended (Dokas et al., 2013). Table 1 shows the STPA and EWaSAP steps.

Table 1. EWaSAP steps as add-ons to STPA

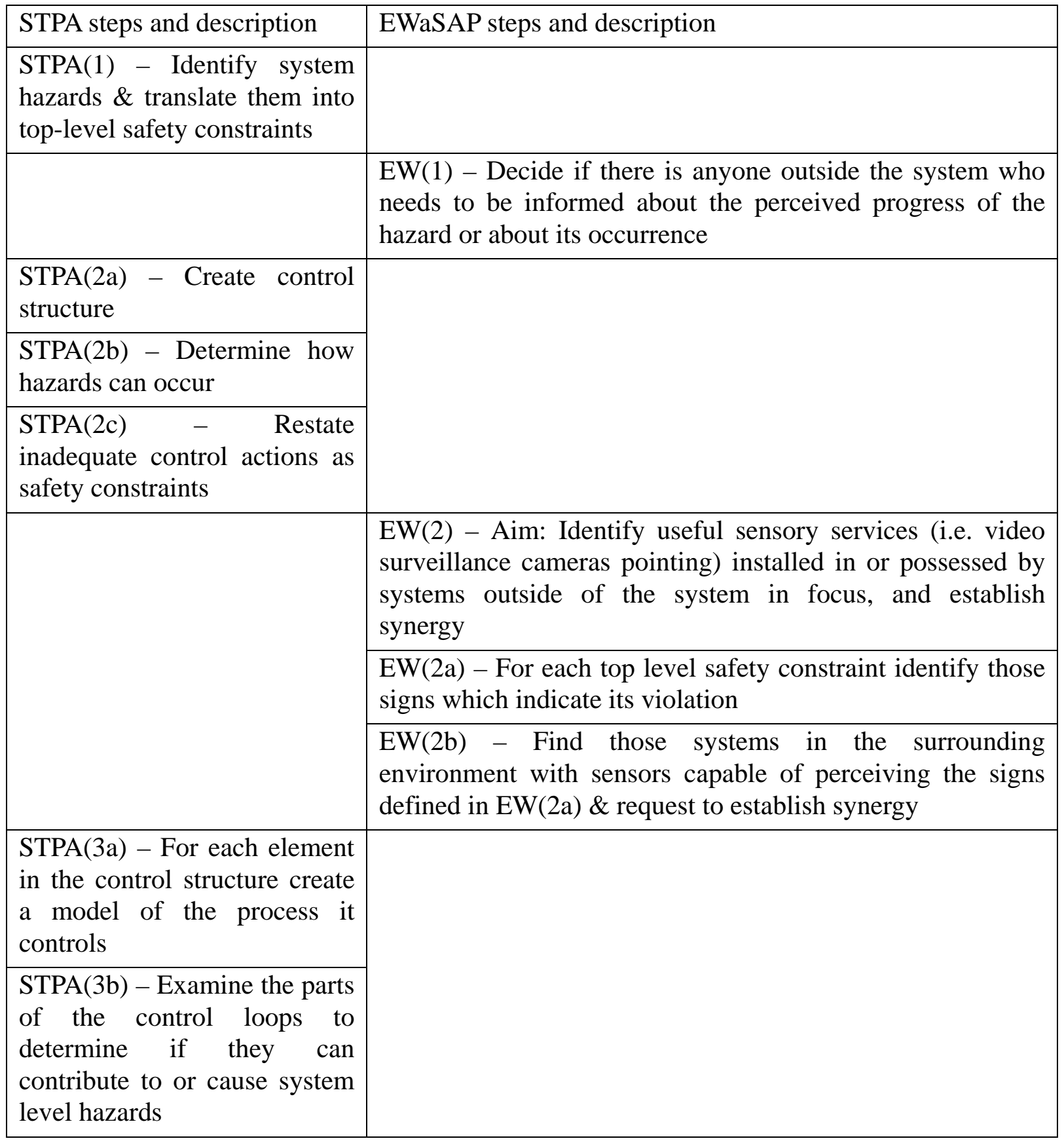




\begin{tabular}{|c|c|}
\hline STPA steps and description & EWaSAP steps and description \\
\hline & EW(3) - Aim: Enforce Internal Awareness Actions \\
\hline & $\begin{array}{l}\text { EW(3a) - Describe what needs to be monitored \& what type } \\
\text { of features/capabilities the sensors must have so that to } \\
\text { make the appropriate controllers capable of perceiving: } \\
\text { - the signs indicating the occurrence of the flaw } \\
\text { - the violation of the assumptions made during the design of } \\
\text { the system }\end{array}$ \\
\hline & $\begin{array}{l}\text { EW(3b) - After design trade-offs and selection of sensors, } \\
\text { define which patterns of perceived data indicate the } \\
\text { occurrence of the flaw and/or the violation of its designing } \\
\text { assumptions }\end{array}$ \\
\hline & $\begin{array}{l}\text { EW(3c) - Update the process models of the controllers with } \\
\text { appropriate awareness and control actions, which should be } \\
\text { enforced based on the perceived early warning signs, so that } \\
\text { to warn about, adapt to, or eliminate the causal factor to the } \\
\text { loss which is present in the system }\end{array}$ \\
\hline & $\begin{array}{l}\text { EW(3d) - For each perceived warning sign, define its } \\
\text { meta-data/attribute values to ensure that it will be perceived } \\
\text { and ultimately understood by the appropriate controller/s }\end{array}$ \\
\hline $\begin{array}{l}\text { STPA(4) - Restate any flaws } \\
\text { identified as safety constraints } \\
\& \quad \text { repeat STPA(3a) \& } \\
\text { STPA(3b) }\end{array}$ & \\
\hline
\end{tabular}

After this step, the real system(s) is produced based on a mapping between itself and the composition of the desired system.

\subsection{Dissimilarity Measures for Binary Data}

In the literature, there are plenty of distance/dissimilarity measures, which aim at detecting the mismatching bits of binary data sets. In this study, Rogers-Tanimoto was chosen as a dissimilarity measure for comparing two vectors each time by giving double weight to the dissimilarities between the compared vectors. In this way, the distance between the vectors is not seen as linear, as suggested by various authors for socio-technical systems (e.g., Leveson, 2011; Brachthaeuser, 2011; Benvenuto 2007), and even a few differences might result to high dissimilarities (e.g. two systems with a vector of 100 points, 50 of which are different, have a dissimilarity of 0.67 , where 1.0 is the maximum value of dissimilarity).

The Rogers-Tanimoto formula is (Zhang and Srihari, 2003):

$$
R T d(i, r)=\frac{2 S 10+2 S 01}{S 11+S 00+2 S 10+2 S 01}
$$

In the formula above, S00 and S11 represent identical properties/values, whereas S01 and S10 correspond to different ones. In general, some facts about dissimilarity measures are the 
following:

(a) The minimum dissimilarity is ' 0 '; that is, the vectors are similar.

(b) All variables are normalised, i.e. between ' 0 ' and ' 1 '.

(c) Distance can be defined as the dual of a similarity measure, i.e. $d\left(b_{v} r\right)-1-s(1, r)$. This literally means that a similarity can be expressed as the complementary of the corresponding dissimilarity, and vice versa.

\subsection{Research Hypothesis}

The hypothesis tested for all three case studies with the RiskSOAP methodology is: "Provided that there are more than one versions of the same system that differ in their composition, the RiskSOAP methodology is adopted and the RiskSOAP indicator is calculated as many times as the different alternative versions of the system. After obtaining these values, it is expected that the lowest ${ }^{1}$ value for the RiskSOAP indicator will be returned for the system version that is proclaimed as less vulnerable, and vice versa."

\section{The 3 Case Studies}

The three case studies described below were used to measure the distance between different system versions with the RiskSOAP.

Case 1: ACROBOTER (Stepan et al., 2009) was a robotic installation aimed to demonstrate a radically new robot locomotion technology that could effectively be used in a home or a workplace environment for manipulating small objects autonomously or in close cooperation with humans. Because the original system failed to meet its purposes and to deliver the tasks as described in the project scope, the designers came up with an updated version. The modified version (i.e. operated system) was enriched with elements that the developers, based on their experience, considered as important.

Case 2: The Überlingen mid-air collision accident occurred in 2002 between Bashkirian Airlines (Russia) and a DHL operated aircraft. The official accident reports (Johnson, 2004; BFU, 2002) involved both technical and organisational deficiencies. In this accident technical system capabilities such as optical STCA ${ }^{2}$, phone connection, TCAS ${ }^{3}$ downlink, etc., and

\footnotetext{
${ }^{1}$ It will be the lowest because instead of focusing on the similarities between the compared system composition versions, the detection of differences is what matters most. The lower the indicator the lower the distance between the examined systems.

2 Short-term conflict alert (STCA) is an automated warning system for air traffic controllers (ATC). It is a ground-based safety net intended to assist the controller in preventing collisions between airborne aircraft by generating, in a timely manner, an alert of a potential or actual infringement of separation minima.

3 A traffic collision avoidance system (TCAS) is installed on aircraft and designed to eliminate mid-air collisions. Independent of air traffic control, it scans the airspace around an aircraft in order to communicate with other aircraft equipped with TCAS and warns pilots about the proximity of other aircraft, which may pose a threat for mid-air collision.
} 
organisational issues, detailed and unified directives, additional aid to the ATC, etc., were either not available or degraded.

Case 3: The examined road tunnel (Chatzimichailidou and Dokas, 2016) is a recent construction of the A23 Greek motorway that connects Greece to Bulgaria. According to the project manager, the tunnel is planned to be renovated in the next couple of years. Its length is $519 \mathrm{~m}$ and it has one tube with bi-directional traffic. Because the tunnel is located in a mountainous area, the speed limit is $60 \mathrm{~km} / \mathrm{h}$ and the percentage of heavy goods vehicles is estimated to be around 20\% of the annual average daily traffic volume (i.e. 1500 vehicles per tube), while lorries that carry dangerous goods are banned from the tunnel. The tunnel is not yet monitored by an exclusive tunnel control centre.

\section{Results}

Due to space limitations, the numerical results of the three case studies are summarised in this section with the goal to test the hypothesis and demonstrate how RiskSOAP can contribute to the design, development, operation and maintenance of safely engineered systems. For a better understanding, a graphical form of the hypothesis is given in Figure 2.

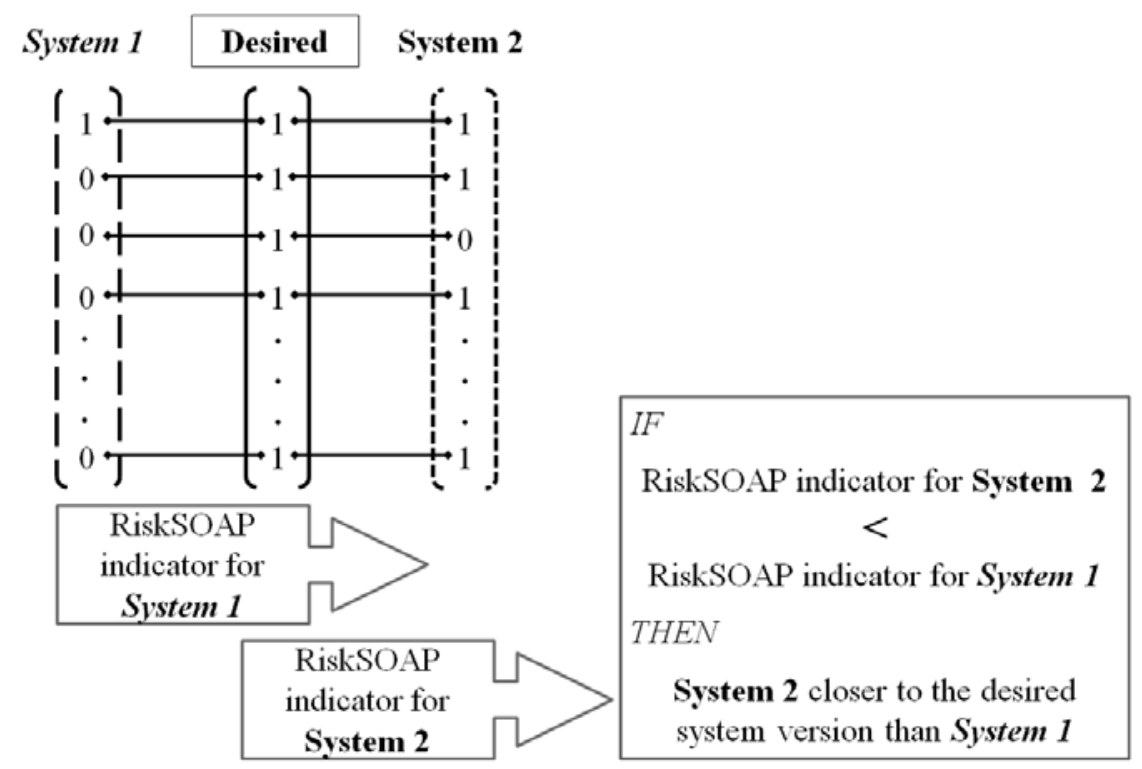

Figure 2. Comparison of the different system versions using the RiskSOAP indicator and condition checked (Chatzimichailidou, 2016)

\subsection{ACROBOTER case}

In this case study, RiskSOAP was used to quantify the distance of ACROBOTER's different versions; (a) the ideal system derived from STPA and EWaSAP (b) the original (i.e. 'as-is') ACROBOTER, and (c) the system as designed ad-hoc. The values derived from the RiskSOAP indicator (Table 2) imply that both system versions do not satisfy the requirements generated with STPA and EWaSAP. Due to space saving reasons, only a few safety requirements and sensor characteristics are given in Figure 3 for the ACROBOTER case. 


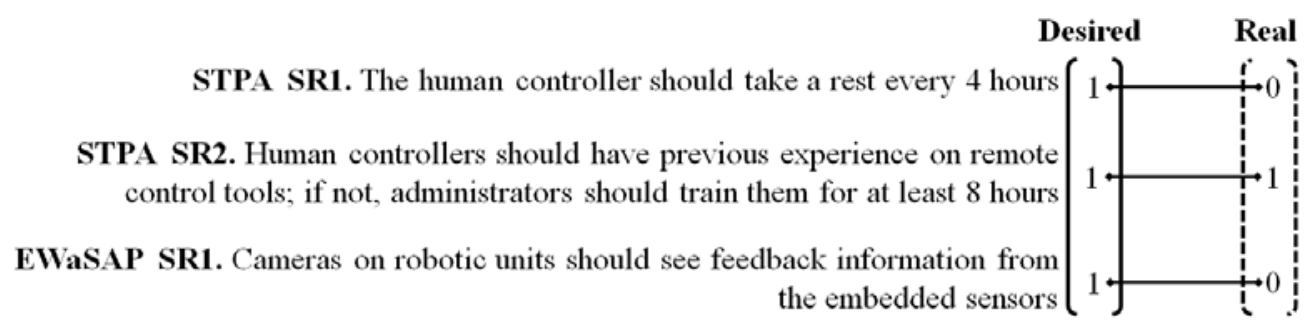

Figure 3. Extraction of binary vectors in the ACROBOTER case

To enhance the safety of ACROBOTER, modifications in the system composition can be considered. For example, the value of the RiskSOAP indicator will decrease by:

(a) imposing critical safety constraints on the system behaviour in order to avoid unsafe events or conditions,

(b) adding appropriate sensors to provide controllers with a high-level overview of the workspace, as well as developing communication channels through which warning messages with rich content will flow, and

(c) fostering appropriate mental and process models on the grounds of mutual understanding and action-taking between system controllers.

Table 2. Numerical results for ACROBOTER (Chatzimichailidou, 2016)

\begin{tabular}{|l|l|l|l|}
\hline $\begin{array}{l}\text { System } \\
\text { Elements }\end{array}$ & $\begin{array}{l}\text { Ideal System } \\
\text { (STPA \& } \\
\text { EWaSAP) }\end{array}$ & Original system & Redesigned system \\
\hline Present & 213 & 96 & 92 \\
\hline Absent & 0 & 117 & 121 \\
\hline Vectors' length & 213 & 213 & 213 \\
\hline RiskSOAP indicator & $\begin{array}{l}=2 * 117+2 * 0 / 96+0+2+2 * 121+2 * 0 / 92+0 \\
2 * 117+2 * 0=0.7091\end{array}$ & \begin{tabular}{l}
$2 * 121+2 * 0=0.7246$ \\
\hline $\begin{array}{l}\text { Difference between the 'as-is' } \\
\text { system versions }\end{array}$
\end{tabular} & $\begin{array}{l}=0.7246-0.7091 \\
=0.0155\end{array}$ \\
\hline
\end{tabular}

With the numerical expression provided by the indicator, the system dynamics are communicated. Namely, by calculating the RiskSOAP indicator every time a change in the system composition is made, the fluctuation in the safety risk SA can be observed. This also means that the RiskSOAP methodology does not apprehend just a snapshot of the system design status.

\section{2 Überlingen mid-air collision case}

In the specific case, the RiskSOAP was used for the following system versions; (a) the ideal system based on STPA and EWaSAP, (b) the original system composition taking into account the regulations that existed prior to the accident, and (c) the system improvements proposed in the official accident reports. As presented in Table 3, the RiskSOAP indicator value 
obtained after comparing the STPA-EWaSAP vector to the operated one was 0.8471, while the value obtained after comparing the STPA-EWaSAP system vector to the one suggested by the German Federal Bureau of Aircraft Accident Investigation (BFU 2002) and Johnson (2004) was equal to 0.6840. A general conclusion to be drawn is that both the operated and the BFU \& Johnson system versions deviate from the ideal STPA-EWaSAP design, since the RiskSOAP indicator is much higher than zero. However, the recommendations made after the safety investigation decreased the value RiskSOAP indicator, meaning that improved system safety.

Table 3. Numerical results for the Überlingen mid-air collision accident (Chatzimichailidou, 2016)

\begin{tabular}{|l|l|l|l|}
\hline System Elements & $\begin{array}{l}\text { Ideal system } \\
\text { (STPA \& } \\
\text { EWaSAP) }\end{array}$ & System before the accident & System after the accident \\
\hline Present & 279 & 74 & 134 \\
\hline Absent & 0 & 205 & 145 \\
\hline Vectors' length & 279 & 279 & 279 \\
\hline \multicolumn{2}{|l|}{ RiskSOAP indicator } & $=0.8471$ & $=0.6840$ \\
\hline Difference & $=0.1631$ & \\
\hline
\end{tabular}

\subsection{Road tunnel fire case}

In this case study, the RiskSOAP quantified the distance of system's different versions; (a) the ideal system in terms of STPA and EWaSAP, (b) the original system composition with the regulations that existed prior to the EU (2004) and PIARC directives (World Road Association, 2007), and (c) the proposed system composition with all the aforementioned directives considered. The RiskSOAP indicator value obtained after comparing the STPA-EWaSAP system vector to the operated one was 0.6007 , while the value obtained after comparing the STPA-EWaSAP vector to the one suggested by the European Directive and PIARC was equal to 0.3319 (Table 4). It is observed that although the system which incorporates the directives is safer that the original (i.e. lower RiskSOAP), still the EU \& PIARC system version does not meet all requirements of the STPA-EWaSAP one.

Table 4. Numerical results for the road tunnel (Chatzimichailidou, 2016)

\begin{tabular}{|l|l|l|l|}
\hline $\begin{array}{l}\text { System } \\
\text { Elements }\end{array}$ & $\begin{array}{l}\text { Ideal } \\
\text { system } \\
\text { (STPA \& } \\
\text { EWaSAP) }\end{array}$ & $\begin{array}{l}\text { Original } \\
\text { system }\end{array}$ & $\begin{array}{l}\text { System incorporating Directive 2004/54/EC } \\
\text { \& PIARC }\end{array}$ \\
\hline Present: & 191 & 109 & 153 \\
\hline Absent: & 0 & 82 & 38 \\
\hline Vector's & 191 & 191 & 191 \\
\hline
\end{tabular}




\begin{tabular}{|l|l|l|l|}
\hline & $\begin{array}{l}\text { Ideal } \\
\text { system } \\
\text { Elements }\end{array}$ & $\begin{array}{l}\text { Original } \\
\text { (STPA \& } \\
\text { EWaSAP) }\end{array}$ & $\begin{array}{l}\text { System } \\
\text { s PIARC }\end{array}$ \\
\hline length: & & & \\
\hline \multicolumn{2}{|l|}{ RiskSOAP indicator } & $=0.6007$ & $=0.3319$ \\
\hline \multicolumn{2}{|l|}{ Difference } & $=0.2688$ & \\
\hline
\end{tabular}

The comparison between the values of the two right columns in Table 4 shows that the suggested by the Directive and PIARC tunnel has more enhanced safety than the original one. Practically, this means that the controllers of the former system may be more capable of perceiving and preventing identified hazards than before.

\section{Discussion}

The hypothesis formulated in section 2.4 above was proven through all three cases, and the validity of the RiskSOAP indicator was demonstrated. The results of the RiskSOAP indicator in the cases of the Überlingen accident and the road tunnel showed that the improved system compositions corresponded to lower values of the indicator. The changes suggested by the BFU (2002) and Johnson (2004) led to a decrease of the RiskSOAP indicator (i.e. lower dissimilarity from the ideal system), compared to the one involved in the accident. Similarly, the originally designed tunnel was more vulnerable compared to the one proposed by the EU and PIARC. Hence, a lower RiskSOAP indicator corresponds to a safer system assuming that the additional system characteristics are effectively operationalised and do not impose side risks to neighbour systems. The distance between the ideal systems generated by STPA and EWaSAP and their upgraded versions for all three cases show that there is still potential for safety improvements even for systems that are currently contemplated as safe enough.

With the numerical expression provided by the RiskSOAP indicator, the differences between system versions are communicated in a simple and understandable manner, thus enhancing the safety risk SA of the system owner. Every time a change in the system's composition is made, the RiskSOAP indicator can be calculated before and after changes take place. Based on the different values of the indicator, their overall fluctuation and the priorities and constraints of the industry, management can act upon system changes.

The steps of the RiskSOAP methodology can be repeated and the RiskSOAP indicator can be recalculated every time changes in the system composition occur. This will allow assessment of how and to what degree changes in the system throughout its lifecycle affect its safety. A predefined threshold of the RiskSOAP indicator can serve as a criterion for evaluating modifications that might affect the minimum acceptable safety level of the system under consideration. Indeed, such a threshold will be based on the available resources, i.e. time, budget, available technology and human operators; however, it can be lowered when circumstances allow and a space for safety improvements is available. 


\section{Conclusions}

The measurement of the RiskSOAP depicted the distance of systems from their ideal composition and showed the potential for further improvements in terms of safety constraints generated through the STPA and EWaSAP methods. The RiskSOAP indicator can serve in regard to safety as (a) a selection criterion between alternative designs of the same system and (b) a decision-making tool when evaluating system changes with reference to the ideal system. The former might be achieved by comparing the vectors corresponding to different design versions; the lower the value of the indicator, the better the system design version in terms of safety embedded. As a decision-making tool, the RiskSOAP indicator can benefit managers and engineers through the consideration of system composition modifications with the goal to shorten the distance between vectors and 'lessen' the dissimilarity between operated and ideal system versions. For systems that have already evolved and have inevitably degraded over time, the 'ideal system architecture' can be used as a benchmark for system modifications.

Regarding the practicality of the RiskSOAP methodology, the performance of the STPA and EWaSAP steps require analysts who are experienced, well qualified and supported by an interdisciplinary team with common and complementary understanding of the system under study. The individual and team skills will determine the inclusiveness of the STPA and, by extension, the results of the RiskSOAP methodology. Moreover, the subjective interpretation of the value of the indicator is inevitable and may differ across systems and designers, affecting the degree of interventions in the systems. In addition, the RiskSOAP binary-based indicator implies that there is no intermediate case lying between absence and presence of system characteristics, neglecting that the variables may have a true value that ranges between the ' 0 ' and ' 1 ' extremes. Thus, the methodology proposed in this paper might be further improved by considering dissimilarity measures which count for intermediate states of system components.

Furthermore, since the STPA analysis is applicable to emergent properties other than safety such as security and quality, and to various business functions such as production and finance, the RiskSOAP methodology can be equivalently adapted to cover the aforementioned areas. For example, if the main purpose of the system is related to economics, then the selection criteria of the system's elements would be based on economic or econometric models, and not on systems safety models.

As a final conclusion, RiskSOAP offers to system agents the opportunity to become aware of deviations of the system from its desired state; and the RiskSOAP methodology paves the path towards the design and operation of safer complex socio-technical systems. The quantification of the differences between original design and current state of a system reflects the extent of violations of the design assumptions and can be seen as a leading safety indicator under the approach proposed by Leveson (2015).

\section{References}

Benvenuto, S. (2007). Simplistic Complexity: A discussion on psychoanalysis and chaos 
theory. World Futures, 61(3), 181-187.

Blandford, A., Furniss, D., \& Vincent, C. (2014). Patient safety and interactive medical devices: realigning work as imagined and work as done. Clinical Risk, DOI: $10.1177 / 1356262214556550$.

Brachthaeuser, C. (2011). Explaining global governance-a complexity perspective. Cambridge Review of International Affairs, 24(2), 221-244.

Chatzimichailidou, M. M. (2016). RiskSOAP: A Methodology for Measuring Systems' Capability of Being Self-Aware of Their Threats and Vulnerabilities (Doctoral thesis). Democritus University of Thrace, Xanthi, Greece. [Online] Available: http://www.didaktorika.gr/eadd/handle/10442/37027?locale=en

Chatzimichailidou, M. M., \& Dokas, I. M. (2016). RiskSOAP: introducing and applying a methodology of risk self-awareness in road tunnel safety. Accident Analysis \& Prevention, 90, 118-127.

Chatzimichailidou, M. M., \& Dokas, I. M. (2015). Introducing RiskSOAP to communicate the distributed situation awareness of a system about safety issues: an application to a robotic system. Ergonomics, 1-14.

Dokas, I. M., Feehan, J., \& Imran, S. (2013). EWaSAP: An early warning sign identification approach based on a systemic hazard analysis. Safety science, 58, 11-26.

European Commission (2004). Directive 2004/54/EC of the European Parliament and of the Council of 29 April 2004 on minimum safety requirements for tunnels in the Trans-European Road Network, OJ L 167 of 30/04/2004 p. 39 corrigendum OJ L 201 of 07/06/2004, p. 56. European Commission, Brussels.

German Federal Bureau of Aircraft Accident Investigation (BFU). (2002). BFU Überlingen Investigation Report. Reference AX001-1-2/02.

Johnson, C.W. (2004). Final Report: review of the BFU Überlingen Accident Report. Contract C/1.369/HQ/SS/04 to Eurocontrol.

Leveson, N. (2015). A systems approach to risk management through leading safety indicators. Reliability Engineering \& System Safety, 136, 17-34.

Leveson, N. (2011). Engineering a safer world: Systems thinking applied to safety. Mit Press.

Woltjer, R., Pinska-Chauvin, E., Laursen, T., \& Josefsson, B. (2015). Towards understanding work-as-done in air traffic management safety assessment and design. Reliability Engineering \& System Safety, 141, 115-130.

World Road Association (PIARC). (2007). Integrated Approach to Road Tunnel Safety. Reference 2007R07EN.

Stepan, G., Toth, A., Kovacs, L. L., Bolmsjo, G., Nikoleris, G., Surdilovic, D., ... \& Kouskouridas, R. (2009, September). ACROBOTER: a ceiling based crawling, hoisting and 


\section{Macrothink}

Journal of Safety Studies

ISSN 2377-3219

2016, Vol. 2, No. 2

swinging service robot platform. In Beyond gray droids: domestic robot design for the 21st century workshop at HCI (Vol. 2009, No. 3, p. 2).

Zhang, B., \& Srihari, S. N. (2003, September). Properties of binary vector dissimilarity measures. In Proc. JCIS Int'l Conf. Computer Vision, Pattern Recognition, and Image Processing (Vol. 1).

\section{Copyright Disclaimer}

Copyright for this article is retained by the author(s), with first publication rights granted to the journal.

This is an open-access article distributed under the terms and conditions of the Creative Commons Attribution license (http://creativecommons.org/licenses/by/3.0/). 